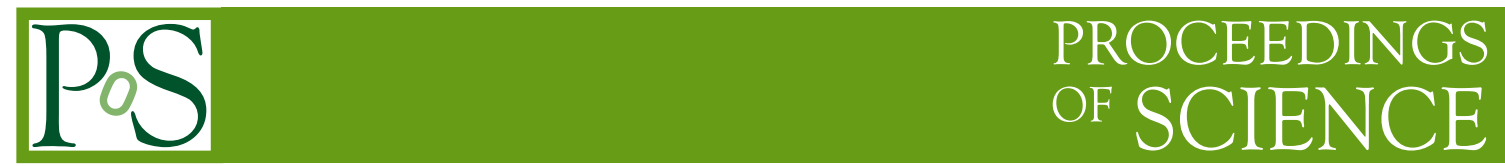

\title{
Recent developments in POWHEG
}

\author{
Paolo Nason* \\ INFN, Sez. di Milano Bicocca \\ E-mail: Paolo.Nason@mib.infn.it
}

I review recent developments in POWHEG, a method for interfacing parton-shower generators with NLO QCD computations. I illustrate recent progress in understanding several features of the method, and in clarifying similarity and differences with respect to MC@NLO. Furthermore, I briefly describe a recently introduced framework, the POWHEG BOX, that allows the automatic POWHEG implementation of any given NLO calculation, and has been recently applied to $Z+$ jet production and to Higgs production via vector-boson fusion.

RADCOR 2009 - 9th International Symposium on Radiative Corrections (Applications of Quantum Field Theory to Phenomenology),

October 25 - 302009

Ascona, Switzerland

${ }^{*}$ Speaker. 
The POWHEG method is a prescription for interfacing NLO calculations with parton shower generators. It was first suggested in ref. [1], and was described in great detail in ref. [2]. Until now, the POWHEG method has been applied to $Z$ pair hadroproduction [3], heavy-flavour production [4], $e^{+} e^{-}$annihilation into hadrons [5] and into top pairs [6], Drell-Yan vector boson production [7, 8], $W^{\prime}$ production [9], Higgs boson production via gluon fusion [10, 11], Higgs boson production associated with a vector boson (Higgs-strahlung) [11], single-top production [12] $Z+1$ jet production [13], and, very recently, Higgs production in vector boson fusion [21]. Unlike MC@NLO [14], POWHEG produces events with positive (constant) weight, and, furthermore, does not depend on the subsequent shower Monte Carlo program. It can be easily interfaced to any modern shower generators and, in fact, it has been interfaced to HERWIG $[15,16]$ and PYTHIA [17] in refs. $[3,4,7,10,12]$.

\section{The concept}

The basic concept of POWHEG is better clarified by considering a process with a single massless coloured parton involved (one can consider, for example, semileptonic top decay, neglecting the $b$ mass). In a shower Monte Carlo, the radiation of a final state light parton is generated with an algorithm that resums all leading log corrections to the Born process. The hardest emission in a shower Monte Carlo is generated according to the formula [1]

$$
d \sigma=B\left(\Phi_{B}\right) d \Phi_{B}\left[\Delta_{t_{0}}^{\mathrm{MC}}\left(\Phi_{B}\right)+\Delta_{t}^{\mathrm{MC}}\left(\Phi_{B}\right) \frac{R^{\mathrm{MC}}(\Phi)}{B\left(\Phi_{B}\right)} d \Phi_{r}^{\mathrm{MC}}\right]
$$

where $t$ is the radiation transverse momentum, $t_{0}$ is the minimum allowed value for $t$ (typically of the order of a hadronic scale), $B\left(\Phi_{B}\right) d \Phi_{B}$ is the Born differential cross section, and $R^{\mathrm{MC}} d \Phi_{B} \Phi_{r}$ is the real radiation differential cross section in the Monte Carlo (MC from now on) approximation. It is assumed that the full phase space including radiation is parametrized in term of the Born phase space $\Phi_{B}$ and the radiation phase space $\Phi_{r}$, i.e. $\Phi=\Phi\left(\Phi_{B}, \Phi_{r}\right)$. In typical MC's, the radiation phase space is determined by three variables characterizing the collinear splitting process, like, for example, the splitting angle, the momentum fraction and the azimuth. The radiation transverse momentum $t$ is a function of $\Phi_{B}$ and $\Phi_{r}$. It can be defined as the momentum component of the radiated parton orthogonal to the momentum of the radiating parton. The MC Sudakov form factor

$$
\Delta_{t_{l}}^{\mathrm{MC}}\left(\Phi_{B}\right)=\exp \left[-\int_{t>t_{l}} \frac{R^{\mathrm{MC}}(\Phi)}{B\left(\Phi_{B}\right)} d \Phi_{r}^{\mathrm{MC}}\right]
$$

represents the probability for not having radiation harder than $t_{l}$.

The basic idea in POWHEG is to improve formula (1.1) in such a way that NLO accuracy is reached. One replaces formula (1.1) with the following one

$$
d \sigma=\bar{B}\left(\Phi_{B}\right) d \Phi_{B}\left[\Delta_{t_{0}}^{s}\left(\Phi_{B}\right)+\Delta_{t}^{s}\left(\Phi_{B}\right) \frac{R^{s}(\Phi)}{B\left(\Phi_{B}\right)} d \Phi_{r}^{\mathrm{MC}}\right]+\left[R(\Phi)-R^{s}(\Phi)\right] d \Phi
$$

where $\Phi$ is the full phase space, with $d \Phi=d \Phi_{B} d \Phi_{r}$, and $R$ is the exact radiation cross section. We require that $R \rightarrow R^{s}$ in the soft and collinear limit, and that $R^{s} \leq R$, so that the last contribution in 
the square bracket of eq. (1.3) is non-negative. The choice $R^{S}=R$ is also possible, and it is quite common. We have also defined

$$
\bar{B}\left(\Phi_{B}\right)=B\left(\Phi_{B}\right)+\left[V\left(\Phi_{B}\right)+\int R^{s}(\Phi) d \Phi_{r}\right],
$$

where $V$ is the virtual NLO correction to the Born process. Notice that soft and collinear singularities in $V$ cancel against those arising from the integral of $R^{s}$ in the square bracket of eq. (1.4). The Sudakov form factor is now

$$
\Delta_{t_{l}}^{\mathrm{s}}\left(\Phi_{B}\right)=\exp \left[-\int_{t>t_{l}} \frac{R^{\mathrm{s}}(\Phi)}{B\left(\Phi_{B}\right)} d \Phi_{r} .\right]
$$

The phase space factorization in the POWHEG formula needs not to match that of any shower Monte Carlo. One only requires that in the soft and collinear limit the full phase space $\Phi$ is related to the Born phase space $\Phi_{B}$ in the correct way, i.e. they are identical in the soft limit once the soft particle is removed, and they are identical in the collinear limit once the collinear particles are merged. The POWHEG formula (1.3) can be viewed as an improvement of the Monte Carlo formula (1.1), such that the Born cross section is replaced with an NLO inclusive cross section, and high transverse momentum radiation is corrected so that it becomes exact at large angles. In fact, for large $t$ the Sudakov form factor becomes 1 , and the POWHEG cross section reduces to

$$
d \sigma=\bar{B} \times \frac{R^{s}}{B} d \Phi+\left[R-R^{s}\right] d \Phi \approx R d \Phi,
$$

since $\bar{B} / B=1+\mathscr{O}\left(\alpha_{s}\right)$. At small $t$ the POWHEG formula becomes equal to that of a standard shower $\mathrm{MC}$, up to higher order terms. However, since by construction

$$
\Delta_{t_{0}}^{\mathrm{s}}+\int \theta\left(t-t_{0}\right) \Delta_{t}^{\mathrm{s}} \frac{R^{s}(\Phi)}{B\left(\Phi_{B}\right)} d \Phi_{r}^{\mathrm{MC}}=1
$$

the POWHEG formula maintains NLO accuracy for integrated (i.e. inclusive) quantities.

Notice that the same formula (1.3) also describes the radiation of the hardest parton in MC@NLO, provided $R^{s}$ is identified with the shower Monte Carlo (i.e. HERWIG's) approximation of the real emission cross section. In fact, in MC@NLO two types of events are generated, called $\mathscr{S}$ and $\mathscr{H}$ events. $\mathscr{S}$ events correspond to the term proportional to $\bar{B}$. In MC@NLO the corresponding underlying Born kinematics is generated with a probability $\bar{B}\left(\Phi_{B}\right) d \Phi_{B}$, while the hardest radiation kinematics is generated by the HERWIG shower algorithm. It was demonstrated in ref. [1] that the hardest radiation in HERWIG corresponds to the factor in square bracket multiplying $\bar{B}$ in eq. (1.3). The $\mathscr{H}$ events correspond instead to the $R-R^{s}$ term in eq. (1.3). However, since $R^{s}$ is now given by the HERWIG shower algorithm, there is no guarantee that the difference $R-R^{s}$ should be positive, and this is why negative weighted events are an essential feature of MC@NLO.

The fact that both in MC@NLO and POWHEG the hardest radiation can be described by a similar formula has allowed a better understanding of the agreement and discrepancies between the two approaches. First of all, one understands why most distributions compare very well in the two schemes (see for example $[3,4,7,12]$ ), since they are both described by a similar formula. A first area of discrepancy has emerged following the work of ref. [18]. In $t \bar{t}$ production, a dip in the rapidity distribution of the hardest jet of $M C @ N L O$ was found, that is not present neither in ALPGEN 

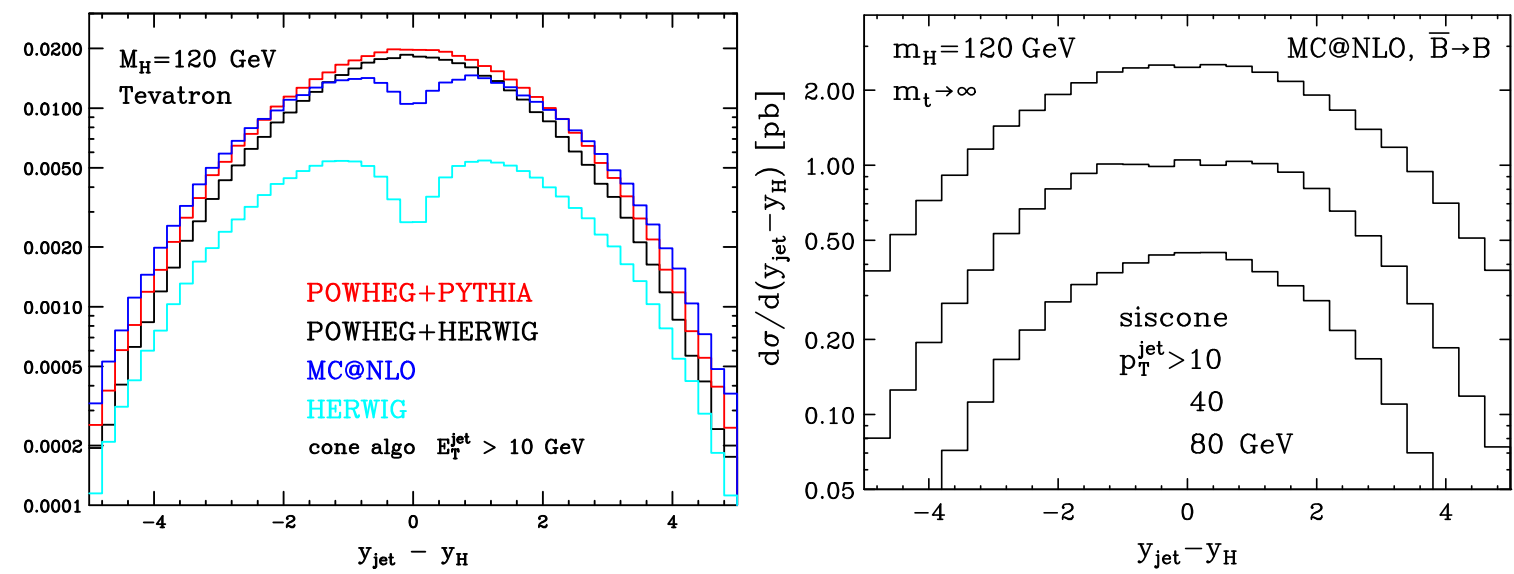

Figure 1: Left: rapidity distribution of the hardest jet relative to the Higgs rapidity. The highest curve at $y=0$ is POWHEG+PYTHIA, next is POWHEG+HERWIG, then MC@NLO and then HERWIG alone. Right: MC@NLO, with $\bar{B}$ replaced by $B$ in the generation of $\mathscr{S}$ events.

nor in POWHEG. It was shown later [7, 10] that this dip is a feature of MC@NLO that is present in several processes. It is particularly visible in Higgs production, as one can see from fig. 1. The pure HERWIG result exhibits a dip at zero rapidity. MC@NLO has a similar dip, although relatively less severe. No dip is present in POWHEG. The origin of the dip is easily understood as a consequence of the presence of the dip in pure HERWIG and of formula (1.3). In fact, for large transverse momenta, formula (1.3) becomes equal to

$$
d \sigma=\bar{B} \times \frac{R^{s}}{B} d \Phi+\left[R-R^{s}\right] d \Phi=R d \Phi+\left[\frac{\bar{B}}{B}-1\right] R^{s} d \Phi .
$$

The second term is small in a perturbative sense, being of order $\alpha_{s}$. However, in the case of Higgs production, where NLO corrections amount to $100 \%$, it is in fact of order 1 . Since $R^{s}$ correspond to the HERWIG approximation to the real cross section, it has a dip that propagates into the MC@NLO cross section. We have checked this explicitly. If this argument is correct, by replacing the $\bar{B}$ cross section with $B$ in MC@NLO, the dip should go away. This is in fact shown in fig. 1, on the right plot.

Another important discrepancy is found in the Higgs $p_{T}$ distribution in the $g g \rightarrow h$ process, displayed in the left plot of fig. 2. At high $p_{T}$ the POWHEG and MC@NLO spectra depart sensibly. The origin of this difference can again be understood starting from formula (1.8). In the POWHEG implementation of Higgs production, the choice $R^{s}=R$ was made, so that formula (1.8) yields

$$
d \sigma=\frac{\bar{B}}{B} \times R d \Phi .
$$

Because of the large Higgs production NLO corrections, the $\bar{B} / B$ factor, instead of being near 1 is close to 2 . Thus NLO corrections amplify the whole $p_{T}$ spectrum in POWHEG. Of course, we could have chosen a different $R^{s}$ in order to reduce this effect, but in view of the plot on the right in fig. 2, this choice was maintained, since the $p_{T}$ spectrum produced in this way is closer to the NNLO result. In MC@NLO this amplification works only for the $\mathscr{S}$ events, that become negligible at large $p_{T}$. Notice that in fig. 2 a mild kink is visible at $80-110 \mathrm{GeV}$ in the MC@NLO curve, signalling the separation of the $\mathscr{S}$ and $\mathscr{H}$ dominated regimes. 

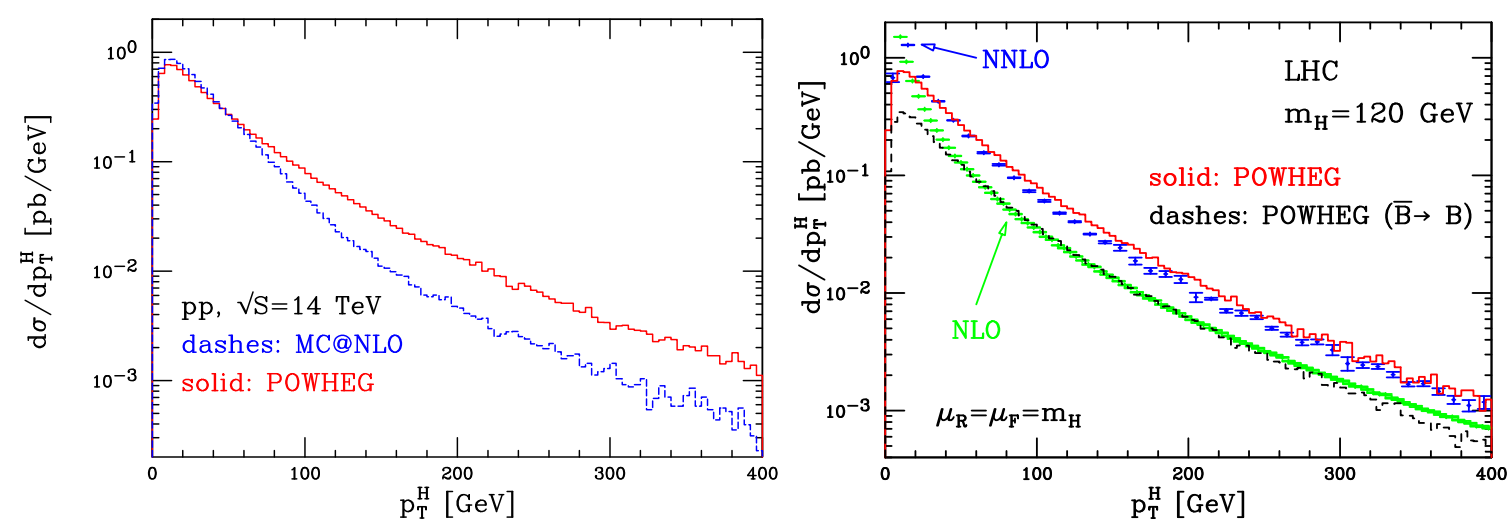

Figure 2: Left: rapidity distribution of the hardest jet relative to the Higgs rapidity. The highest curve at $y=0$ is POWHEG+PYTHIA, next is POWHEG+HERWIG, then MC@NLO and then HERWIG alone. Right: MC@NLO, with $\bar{B}$ replaced by $B$ in the generation of $\mathscr{S}$ events.

\section{The POWHEG BOX}

In ref. [2], the general formulation of POWHEG for complex processes was given, dealing with all complications having to do with the presence of several massless coloured lines, both in the final and in the initial state. In this case formula (1.3) should be applied to each radiating line. What a regular shower Monte Carlo does, starting from a Born process, is to let each coloured massless line radiate more light partons. Within POWHEG one does something similar. However, since in this case the radiation cross section is the exact cross section, rather than an Altarelli-Parisi approximation to it, we work the other way around, decomposing the full real cross section into a sum of terms, each of them singular in only one collinear region. We then adopt a different phase space parametrization $d \Phi \rightarrow d \Phi_{B} d \Phi_{r}$ for each singular region. After this is done, radiation is generated from each collinear region, but only the hardest radiation is retained, and the event is generated according to its kinematics. This procedure reaches a considerable level of complexity, so we decided to implement it in a general way, in order to ease the POWHEG implementation of complex processes. This project, named the POWHEG BOX has been completed [20], and will soon been published. It has been used to reproduce the previous implementation of $W / Z$ and single top production. Furthermore, it has been used to implement $Z+$ jet production [13], and Higgs production in vector boson fusion [21]. This last two processes are the first ones not already present in MC@NLO.

Internally, the POWHEG BOX implements the NLO corrections using the FKS subtraction framework $[22,23]$. The author of an NLO calculation, willing to implement it into the POWHEG BOX framework, needs only to supply the following ingredients: a) the Born phase space; b) the list of Born and Real processes in a specified format; c) the Born squared amplitudes $B=\left|\mathscr{M}^{2}\right|$, the colour correlated one $B_{i j}$, and the spin correlated one $B_{j, \mu_{j}, \mu_{j}^{\prime}}$, for each Born subprocess (these ingredient are typically needed in NLO calculations performed with the subtraction method, and, for example, are generated automatically in the MadDipole package [24]); d) the real squared amplitude, for all relevant partonic processes (this is easily obtained from programs like MadGraph [25]); e) the finite part of the virtual amplitude for all relevant partonic processes, which is the only 
ingredient that may require dedicated work. The POWHEG BOX does all the rest, including the automatic implementation of the FKS subtractions.

\section{Conclusions}

Much progress has been achieved in POWHEG in this past year. Several features of the method have been better understood in simple terms, and its comparison with MC@NLO has been considerably clarified. An important step towards automation has been achieved with the construction of the POWHEG BOX. Thanks to this framework, a relatively complex process like VBF Higgs production has been implemented in a very short time. It is our hope that the POWHEG BOX will allow authors of NLO calculations to implement their work in the POWHEG framework with a limited effort.

\section{Acknowledgements}

All results presented in this talk have been obtained in collaboration with S. Alioli, C. Oleari and E. Re.

\section{References}

[1] P. Nason, A new method for combining NLO QCD with shower Monte Carlo algorithms, JHEP 11 (2004) 040, [hep-ph / 0409146$].$

[2] S. Frixione, P. Nason, and C. Oleari, Matching NLO QCD computations with Parton Shower simulations: the POWHEG method, JHEP 11 (2007) 070, [arXiv: 0709.2092].

[3] P. Nason and G. Ridolfi, A positive-weight next-to-leading-order Monte Carlo for Z pair hadroproduction, JHEP 08 (2006) 077, [hep-ph / 0606275 ].

[4] S. Frixione, P. Nason, and G. Ridolfi, A Positive-Weight Next-to-Leading-Order Monte Carlo for Heavy Flavour Hadroproduction, JHEP 09 (2007) 126, [arXiv : 0707 . 3088].

[5] O. Latunde-Dada, S. Gieseke, and B. Webber, A positive-weight next-to-leading-order Monte Carlo for $e^{+} e^{-}$annihilation to hadrons, JHEP 02 (2007) 051, [hep-ph/ 0612281$]$.

[6] O. Latunde-Dada, Applying the POWHEG method to top pair production and decays at the ILC, Eur. Phys. J. C58 (2008) 543-554, [arXiv: 0806 .4560].

[7] S. Alioli, P. Nason, C. Oleari, and E. Re, NLO vector-boson production matched with shower in POWHEG, JHEP 07 (2008) 060, [arXiv: 0805 . 4802].

[8] K. Hamilton, P. Richardson, and J. Tully, A Positive-Weight Next-to-Leading Order Monte Carlo Simulation of Drell-Yan Vector Boson Production, JHEP 10 (2008) 015, [arXiv: 0806.0290 ].

[9] A. Papaefstathiou and O. Latunde-Dada, $N L O$ production of $W^{\prime}$ bosons at hadron colliders using the MC@NLO and POWHEG methods, JHEP 07 (2009) 044, [0 901.3685$].$

[10] S. Alioli, P. Nason, C. Oleari, and E. Re, NLO Higgs boson production via gluon fusion matched with shower in POWHEG, JHEP 04 (2009) 002, [arXiv: 0812 . 0578].

[11] K. Hamilton, P. Richardson, and J. Tully, A Positive-Weight Next-to-Leading Order Monte Carlo Simulation for Higgs Boson Production, JHEP 04 (2009) 116, [arXiv: 0903.4345 ].

[12] S. Alioli, P. Nason, C. Oleari, and E. Re, NLO single-top production matched with shower in POWHEG: s- and t-channel contributions, JHEP 09 (2009) 111, [0 907 . 4 076]. 
[13] S. Alioli, P. Nason, C. Oleari, and E. Re. To appear soon.

[14] S. Frixione and B. R. Webber, Matching NLO QCD computations and parton shower simulations, JHEP 06 (2002) 029, [hep-ph/ 0204244$].$

[15] G. Corcella et al., HERWIG 6: An event generator for hadron emission reactions with interfering gluons (including supersymmetric processes), JHEP 01 (2001) 010, [hep-ph/ 0011363 ].

[16] G. Corcella et al., Herwig 6.5 release note, hep-ph/0210213.

[17] T. Sjostrand, S. Mrenna, and P. Skands, Pythia 6.4 physics and manual, JHEP 05 (2006) 026, [hep-ph/0603175].

[18] M. L. Mangano, M. Moretti, F. Piccinini and M. Treccani, Matching matrix elements and shower evolution for top-quark production in hadronic collisions, JHEP 0701 (2007) 013 [hep-ph/0 611129].

[19] S. Alioli, P. Nason, C. Oleari, and E. Re. in preparation.

[20] S. Alioli, P. Nason, C. Oleari, and E. Re, in preparation.

[21] P. Nason and C. Oleari, NLO Higgs boson production via vector-boson fusion matched with shower in POWHEG, arXiv:0911.5299 [hep-ph].

[22] S. Frixione, A General approach to jet cross-sections in QCD, Nucl. Phys. B 507 (1997) 295 [arXiv:hep-ph/9706545 [hep-ph]].

[23] Rikkert Frederix, these proceedings.

[24] R. Frederix, T. Gehrmann and N. Greiner, Automation of the Dipole Subtraction Method in MadGraph/MadEvent, JHEP 0809 (2008) 122 [arXiv: 0808.2128 [hep-ph] ].

[25] J. Alwall et al., MadGraph/MadEvent v4: The New Web Generation, JHEP 0709 (2007) 028 [arXiv:0706.2334 [hep-ph]]. 